\title{
The magnitude of heating of the Diffuse lonized Gas of the interstellar medium by Landau damping of turbulence
}

\author{
S. R. Spangler \\ Department of Physics and Astronomy, University of Iowa, Iowa City, Iowa 52242, USA \\ Received 21 March 2003 / Accepted 27 May 2003

\begin{abstract}
I consider the dissipation of interstellar magnetohydrodynamic turbulence by Landau damping, and the associated heating of the interstellar gas, as a function of the wave distribution function and the plasma $\beta$. The paper particularly addresses the contention of Lerche \& Schlickeiser (2001, LS) that previous treatments of these features were incorrect, and produced expressions which were substantially in error, leading to qualitatively incorrect estimates for turbulent heating of the interstellar medium. Lerche and Schickeiser are correct in noting that the exact expression for the heating rate by dissipation through Landau damping requires integration of the product $2 \gamma_{d} P_{B}$ over all wavenumber space, where $\gamma_{d}$ is the fast magnetosonic wave damping rate, and $P_{B}$ is the magnetic turbulence spatial power spectrum. However, this exact expression yields numerical results which differ little from the previously used expressions, when evaluated for the same turbulence properties. If turbulence in the Diffuse Ionized Gas (DIG) phase of the interstellar medium consisted of fast mode magnetosonic waves, the heat input due to dissipation would exceed the cooling rate by at least 2 to 3 orders of magnitude. This fact may be demonstrated by the formulas in LS as well as previous papers on this topic. Lowering the theoretical rate for heat input requires rather extreme assumptions about the nature of this turbulence, such as very large and seemingly improbable anisotropy factors.
\end{abstract}

Key words. ISM: HII regions - turbulence - plasmas

\section{Introduction}

This paper will deal with theoretical estimates of the heating of the Diffuse Ionized Gas (DIG) component of the interstellar medium by dissipation of magnetohydrodynamic turbulence which resides in the DIG. The Diffuse Ionized Gas is a phase of the interstellar medium characterized by a gas density of about $0.1 \mathrm{~cm}^{-3}$ and a temperature of order 8000-10000 K (Cox \& Reynolds 1987; Haffner et al. 1999). Plasma turbulence is inferred to exist in that medium by radio interstellar scintillations (ISS). The damping of this turbulence will provide heat addition to the interstellar fluid, and it is of interest to compute the volumetric heating rate of this process and compare it with the radiative cooling rate of such gas.

To carry out such a calculation, it is necessary to adopt a model for this interstellar MHD turbulence. One which has been adopted in the past is that of an ensemble of fast mode magnetosonic waves, a plasma phenomenon observed to exist in the solar wind. Further comments on this choice of a model are given in Sect. 2 below. In that case, a prominent damping mechanism is Landau damping, when the waves are obliquely propagating. In previous papers (Spangler 1991; Minter \& Spangler 1997; hereafter Papers I and II) we found that the energy input by damping of the interstellar magnetosonic turbulence was far greater (by orders of magnitude) than the cooling

\footnotetext{
* e-mail: steven-spangler@uiowa.edu
}

rate, provided that "reasonable" turbulence parameters were adopted. These results supported those of previous workers in this area (McIvor 1977; Cesarsky 1980).

The calculations in Papers I and II were recently criticized by Lerche \& Schlickeiser (2001) (hereafter LS). The specific criticisms may be enumerated as follows.

1. LS contend that the use of a reduced power spectrum (i.e. integrated over directions perpendicular to the direction of wave propagation) before carrying out the damping calculation produced significant errors in the turbulence damping rate.

2. It was implied that this method of calculation gave a result for $\epsilon$, the volumetric heating rate, that was in error by a large factor.

3. It was contended that when the heating rate due to Landau damping is correctly calculated, the heating rate due to damping of fast mode turbulence is quite comparable to the cooling rate of the gas, thus suggesting that fast mode waves play a crucial role in the thermodynamics of the interstellar gas.

These three points are summarized by the text in Sect. 5.3 of LS.

In discussing the role of fast magnetosonic waves (for purposes of this paper assumed to be the dominant component of interstellar turbulence) in interstellar thermodynamics, 
it should be stressed that the previous estimates of the heating rate exceed the cooling capacity of the DIG by orders of magnitude. For Landau damping to be much lower, and comparable to the DIG cooling capacity, one of three things has to be the case. (1) The previous formulas used to compute this damping had to have been grossly wrong. (2) The fast mode waves in the interstellar medium must have special properties. This possibility was recognized and discussed in Spangler (1991). (3) The turbulence in the DIG does not consist of fast mode waves, which in fact must comprise a very small fraction of the turbulent energy density. This was the resolution preferred in Papers I and II. To belabor point (1) immediately above, highly precise calculations are not the issue here. We do not know enough about the interstellar turbulence to utilize such expressions.

The response of this paper to the aforementioned points of LS is as follows.

1. Lerche and Schlickeiser are completely correct in noting that the exact expression for the turbulent dissipation rate includes integrating the $\boldsymbol{k}$-dependent damping rate over wavenumber space. Future investigations in this area should carry out the calculation as discussed by LS, or as described in Sect. 4 below.

2. However, the expression used in Spangler (1991) and Minter \& Spangler (1997) (Eq. (6) or (7)) is an entirely satisfactory approximation for the model of turbulence employed in those papers (see Sect. 2 below), so the formulas presented there remain sound.

3. The main correction introduced by carrying out the calculation exactly as described by LS rather than with the approximation of Paper I is the presence of an additional term, describing dissipation and heating even when the net wave propagation angle is zero. Thus, the more exact expression yields a higher heating rate than was previously considered, thus exacerbating the problem with a significant fast mode population in the interstellar turbulence.

4. Heating rates calculated with either the expressions of Papers I and II, corrected versions of those expressions, or the expressions of LS all yield heating rates which are in good agreement, and far higher than the cooling capacity of the DIG, provided that plausible estimates of the interstellar turbulence characteristics are chosen.

5. To obtain approximate parity between the dissipation rate and cooling rate, extreme and seemingly implausible turbulence properties are required. In Papers I and II this large potential reduction in the heating rate was achieved by assuming that the fast mode waves are propagating nearly exactly parallel to the large scale field, or that the ion and electron temperatures were sufficiently low as to result in negligible Landau damping. In LS the dissipation and heating is "turned off" by postulating extreme anisotropy of the turbulence spectrum, to a degree not seen in other natural turbulence fields, and a precise alignment of the turbulence spectrum with respect to the large scale field (parallel propagation).

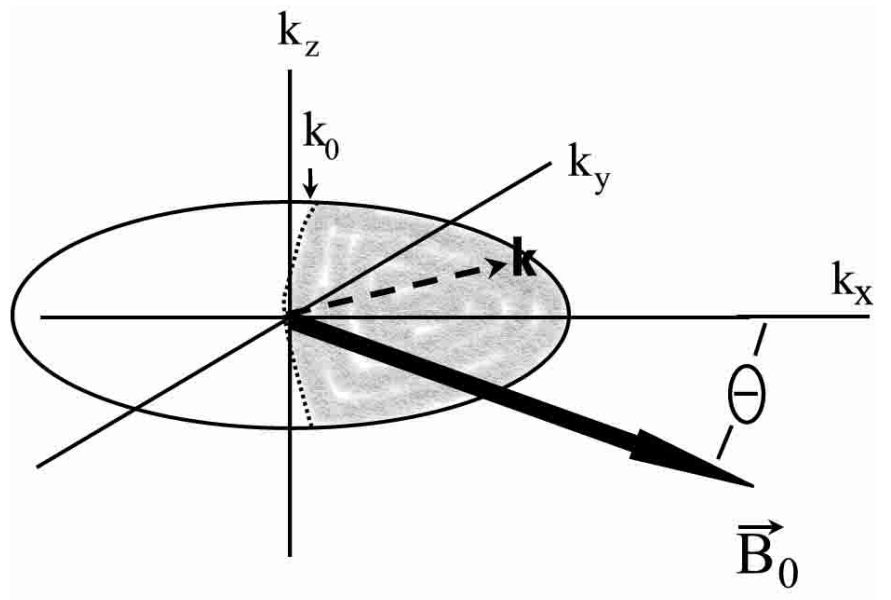

ANISOTROPIC FAST MODE SPECTRUM

Fig. 1. Model of the spectrum of fast mode waves used in the calculations of Papers I and II, and here. The expression for the spectrum is given in Eq. (1). It is particularly to be noted that the propagation direction, $x$, does not coincide with that of the large scale magnetic field, but is inclined at an angle $\Theta$.

\section{Model of the interstellar fast mode spectrum}

The model of the interstellar MHD turbulence power spectrum assumed in Papers I and II is illustrated in Fig. 1. It is shown in this figure, and discussed in this section, because there were misunderstandings about the nature of the assumed spectrum in LS, specifically about the nature of the propagation angle $\Theta$. It was made explicit in Paper I that these waves are modeled on those found upstream of the Earth's bow shock (Russell et al. 1971; Hoppe et al. 1981; Spangler et al. 1988). The angle $\Theta$ was and is intended to represent a systematic obliquity of the wave field, as is observed for the upstream waves, in which case $\Theta$ typically ranges from nearly 0 to $25^{\circ}$ to $30^{\circ}$. It is rarely the case that these waves are perfectly parallel propagating. Furthermore, spacecraft data can be subjected to a minimum variance analysis, which shows that the wave fields do indeed have a systematic angle of propagation with respect to the large scale field. Therefore the angle $\Theta$ remains an important parameter of the turbulence, and not an angle to be integrated over, as stated in LS.

The mathematical form of the spatial power spectrum which we choose is Eq. (1), taken directly from Spangler (1991). This is a generalization of the isotropic power law spatial power spectrum which is often used to model turbulence in the interstellar medium to the case of anisotropic turbulence. The model spectrum in (1) has been used in the interpretation of ISS observations which indicate anisotropy (e.g. Spangler \& Cordes 1998). It is worth noting that an alternative, and perhaps better, spectral model would be one in which equal-power contours lay on the surface of a cone rather than an ellipse, as assumed here. Comments on the consequences of such a model will be given in Sect. 4 below.

It should also be noted that the lower wavenumber limits in this spectrum are such that the power spectral density $=0$ for $k_{x}<k_{\mathrm{o}}$. For $k_{x}>k_{\mathrm{o}}$, the perpendicular wavenumbers can range from 0 to $\infty$. This choice is motivated by the example 
of the waves upstream of the Earth's bow shock, in which the spectrum in the direction of propagation is determined by an ion beam resonance, with a characteristic resonant wavenumber, while the transverse spectrum describes the modulation of the waves in the direction perpendicular to propagation.

It could be argued that the wave fields upstream of the Earth's bow shock (and other shocks in the solar system) are a naive model for the turbulence in the interstellar medium. This may well be the case. However, these fast mode wave fields have been extensively observed and studied, and are known to exist, at least in those heliospheric settings. They also have properties which are in good agreement with theoretical descriptions of linear and nonlinear magnetohydrodynamic waves. In my opinion it is certainly worthwhile examining the consequences should interstellar turbulence consist of such waves, or even have a dynamically significant contribution from them.

\section{Approximate and exact calculations of dissipation rate}

The point of this section is to illustrate the criticism of LS in our earlier calculation, and show the form of the more exact expression.

The model for the spatial power spectrum of the turbulence is

$P_{B}(\boldsymbol{k})=\frac{C_{B}^{2}}{\left[k_{x}^{2}+\Lambda^{2}\left(k_{y}^{2}+k_{z}^{2}\right)\right]^{\delta}}$.

The index $\delta \equiv 1+s / 2$, with $s$ being the index of the omnidirectional power spectrum, which has a value of $5 / 3$ for a Kolmogorov spectrum.

This spectrum was used in Papers I and II as well as Lerche $\&$ Schlickeiser (2001), with the following proviso. In the denominator of the right hand side, I employ $\Lambda^{2}$, whereas LS write $\Lambda$. The $\Lambda^{2}$ convention was used in Papers I and II, for the reason that it is employed in analyses of radio scattering data (e.g. Spangler \& Cordes 1998 and references therein). For such a power spectrum, the anisotropy of the turbulence-broadened image of a radio source will have an axial ratio $\Lambda$ (Narayan \& Hubbard 1988), so the convention here is directly related to observable parameters.

The case discussed here, and presented in Fig. 1, of nearly plane waves with a modulation scale in directions perpendicular to the propagation direction much greater than the modulation scale in the direction of propagation (the so-called "pancake" geometry) corresponds to $\Lambda \gg 1$. The remark to the contrary in LS (section heading for Sect. 4.3.1 of that paper) is incorrect.

As noted above, our approach in Paper I was to model interstellar turbulence in terms of the large amplitude MHD waves upstream of the Earth's bow shock. Some success has been achieved in explaining properties of these waves in terms of one dimensional nonlinear wave equations (the Derivative Nonlinear Schrödinger Equation, Spangler 1997), so in Paper I the interstellar spectrum was replaced by an equivalent $1 \mathrm{D}$ power spectrum, termed the reduced power spectrum, defined as

$\bar{P}_{B}\left(k_{x}\right)=\int_{-\infty}^{+\infty} \int_{-\infty}^{+\infty} \mathrm{d} k_{y} \mathrm{~d} k_{z} P_{B}(\boldsymbol{k})$

which for spectrum (1) is

$\bar{P}_{B}\left(k_{x}\right)=\frac{2 \pi C_{B}^{2}}{s \Lambda^{2}} k_{x}^{-s}$.

The relationship between $\bar{P}_{B}\left(k_{x}\right), C_{B}^{2}$, and $b_{\mathrm{T}}$, the root-meansquare fluctuating magnetic field, is

$b_{\mathrm{T}}^{2}=\int_{k_{\mathrm{o}}}^{k_{\mathrm{i}}} \mathrm{d} k_{x} \bar{P}_{B}\left(k_{x}\right)=\frac{2 \pi C_{B}^{2}}{s(s-1) \Lambda^{2}} k_{\mathrm{o}}^{-(s-1)}$

valid when $s>1$. It should be noted that, in general, one should distinguish between Eq. (4) for the individual components of the fluctuating magnetic field, $b_{\mathrm{T} i}$ and $C_{B i}^{2}$, and those of the total fluctuating field (quadratic sum of the variances in the components) $b_{\mathrm{T}}$ and the corresponding quantity $C_{B}^{2}$. This distinction is made in Minter \& Spangler (1996) and Paper II.

The conceptual simplicity of this approach is that for purposes of damping calculations, the spectrum in Fig. 1 is replaced by plane waves of intensity $\delta b^{2}(k)=\bar{P}_{B}(k) \Delta k$, propagating at an angle $\Theta$ with respect to the large scale magnetic field. This is clearly an approximation to the true damping rate, as was stated on p. 544 of Spangler (1991), and should be increasingly accurate as $\Lambda \rightarrow \infty$.

The expression for the damping rate of plane magnetosonic waves, with a wavevector of magnitude $k$ propagating at an angle $\theta$ with respect to the large scale magnetic field is

$\gamma_{d}=\sqrt{\frac{\pi}{8}} V_{\mathrm{A}} k \frac{\sin ^{2} \theta}{\cos \theta} F(\beta)$.

This equation was used by Papers I and II and LS. This expression is taken from Ginzburg (1961). The function $F(\beta)$ is a shorthand notation introduced in Paper I to describe the dependence of the damping rate on the electron and ion temperatures. Further discussion of this function is given in Sect. 6.

The simplification used in Paper I was to calculate the wave dissipation rate to be the integration (over $k_{x}$ ) of the integrand consisting of the product of (5) and the reduced power spectrum (3), i.e.

$\epsilon_{I}=\frac{1}{4 \pi} \int_{k_{\mathrm{o}}}^{k_{\mathrm{i}}} \mathrm{d} k_{x} 2 \gamma_{d} \bar{P}_{B}\left(k_{x}\right)$.

The convenience of this simplification is that the propagation angle $\Theta$ appears as a parameter rather than a variable of integration.

Substitution of (3) and (5) into (6), and the assumption that the angle of propagation $\Theta$ is small so that $\sin \Theta \simeq$ $\Theta$ and $\cos \Theta \simeq 1$ gives Eq. (15) of Paper $\mathrm{I}$

$\epsilon_{I}=1.2 \times 10^{-20} \Theta^{2} F(\beta) V_{\mathrm{A}}^{\prime} b_{\mathrm{T}}^{\prime 2}\left(l_{\mathrm{o}}^{2} l_{\mathrm{i}}^{\prime}\right)^{-1 / 3} \mathrm{ergs} / \mathrm{s} / \mathrm{cm}^{3}$.

The primed, dimensionless variables are related to the physical variables as follows: the Alfvén speed, $V_{\mathrm{A}}=10^{6} V_{\mathrm{A}}^{\prime} \mathrm{cm} / \mathrm{s}$, the root-mean-square fluctuating turbulent magnetic field 
$b_{\mathrm{T}}=10^{-6} b_{\mathrm{T}}^{\prime}$ Gauss, the outer scale $l_{\mathrm{o}}=\frac{2 \pi}{k_{\mathrm{o}}}=10^{17} l_{\mathrm{o}}^{\prime} \mathrm{cm}$, and the inner scale $l_{\mathrm{i}}=\frac{2 \pi}{k_{\mathrm{i}}}=10^{8} l_{\mathrm{i}}^{\prime} \mathrm{cm}$. These are the dedimensionalizations used in Paper I. This formula was used in Paper I, and with some change in parameters, in Paper II.

Lerche \& Schlickeiser criticized this approach and the resultant Eq. (7), noting that the integration over wavenumber space should include the integration over all wavenumber space, as opposed to the operation here, which is to perform the integration over $k_{y}$ and $k_{z}$ to obtain the reduced spectrum, then multiply by the growth rate and integrate over the wavenumber in the direction of propagation, $k_{x}$.

The Lerche and Schlickeiser point is correct for the most likely model of magnetosonic wave turbulence. In this case, each Fourier component $\boldsymbol{k}$ propagates with the linear dispersion relation of fast magnetosonic waves. The reason for a qualification on this seemingly obvious point is that if one deals with a nonlinear wave, the various Fourier components do not propagate independently of each other with the linear dispersion relation, and the approach of Paper I might be physically accurate as well as being a computational simplification. Nonetheless, in what follows, I will calculate the dissipation/heating rate with the wavenumber integration described by LS.

In this case, the dissipation rate is

$$
\epsilon_{N}=\frac{1}{4 \pi} \int_{V_{k}} \mathrm{~d}^{3} k 2 \gamma(\boldsymbol{k}) \frac{C_{B}^{2}}{\left[k_{x}^{2}+\Lambda^{2}\left(k_{y}^{2}+k_{z}^{2}\right)\right]^{\delta}}
$$

with $\gamma(\boldsymbol{k})$ given by Eq. (5), in which $\theta$ is a variable of integration which must be included in the integration in (8). The integration is over $V_{k}$, the volume in $\boldsymbol{k}$ space in which the waves exist (Fig. 1).

The primary issue in this paper is the magnitude of the difference involved in calculating the dissipation rate via (7) as opposed to (8). LS contend that the difference is large enough to qualitatively change conclusions about the thermodynamics of the interstellar medium. The use of Eq. (2) through Eq. (6) as a valid approximation, as assumed in Paper I, on the other hand, would require that the differences be relatively small, i.e. within a factor of order unity. I would again emphasize that previous estimates of the Landau damping heating rate in the interstellar medium exceed the cooling rate by orders of magnitude. To be astrophysically significant, the differences in the formulas would have to be similarly great.

\section{Revised dissipation rate calculation}

In this section, we evaluate the expression (8) for the case of the magnetosonic wave spectrum shown in Fig. 1. We first change to a cylindrical coordinate system with the axial direction being $x$, and defining $\left.k_{\perp}=\sqrt{(} k_{y}^{2}+k_{z}^{2}\right)$,

$$
\begin{aligned}
\epsilon_{N}= & \frac{C_{B}^{2}}{2 \pi} \sqrt{\frac{\pi}{8}} F(\beta) V_{\mathrm{A}} \int_{k_{\mathrm{o}}}^{k_{\mathrm{i}}} \mathrm{d} k_{x} \int_{0}^{\infty} \mathrm{d} k_{\perp} \\
& \times \int_{0}^{2 \pi} \mathrm{d} \phi \frac{k_{\perp} \sqrt{\left(k_{x}^{2}+k_{\perp}^{2}\right) \sin ^{2} \theta}}{\left[k_{x}^{2}+\Lambda^{2} k_{\perp}^{2}\right]^{\delta}}
\end{aligned}
$$

In the case of Eq. (9) $\theta$ is the angle between a given Fourier component $\boldsymbol{k}=\left(k_{x}, k_{\perp}, \phi\right)$ and the large scale field. It is a variable which partakes in the integration, and is not to be confused with the systematic propagation angle $\Theta$ (see Fig. 1) which is the angle between $\boldsymbol{B}_{0}$ and the $x$ axis.

Two similar approximations have been made leading to (9), to facilitate the simplified analytic expression given below (12). This first is that $\gamma_{d} \propto \sin ^{2} \theta$, thus approximating $\cos \theta \simeq 1$. This approximation will be accurate for large anisotropy factors and small systematic propagation angles $\Theta$. It may be seen from Eq. (5) that this approximation will underestimate $\gamma_{d}$ and thus $\epsilon_{N}$, if contributions from oblique modes are significant. The second approximation is that the $\theta$ dependence in $F(\beta)$ (seen in Eq. (23) below) has similarly been ignored, so that the damping rate may be factored as in Eq. (5), so $F(\beta)$ does not partake of the angular integrations. This point is considered further in Sect. 6.

The relationship between $\theta$ and the $\boldsymbol{k}$-space variables is as follows. Let $\xi$ be the angle between a mode $\boldsymbol{k}$ and the $x$ axis, $\tan \xi=k_{\perp} / k_{x}$. Then it may be shown, using spherical trigonometry relations, that

$$
\begin{aligned}
\sin ^{2} \theta= & 1-\cos ^{2} \xi \cos ^{2} \Theta-\sin ^{2} \xi \sin ^{2} \Theta \cos ^{2} \phi \\
& -2 \cos \xi \cos \Theta \sin \xi \sin \Theta \cos \phi .
\end{aligned}
$$

If identity (10) is substituted into (9), the $\phi$ integrations are simple, and the last term vanishes. The resultant expression is

$$
\begin{aligned}
\epsilon_{N}= & \sqrt{\frac{\pi}{8}} C_{B}^{2} F(\beta) V_{\mathrm{A}} \int_{k_{\mathrm{o}}}^{k_{\mathrm{i}}} \mathrm{d} k_{x} \int_{0}^{\infty} \mathrm{d} k_{\perp} \frac{\left.k_{\perp} \sqrt{(} k_{x}^{2}+k_{\perp}^{2}\right)}{\left[k_{x}^{2}+\Lambda^{2} k_{\perp}^{2}\right]^{\delta}} \\
& \times\left(1-\cos ^{2} \xi \cos ^{2} \Theta-\sin ^{2} \xi \sin ^{2} \Theta / 2\right)
\end{aligned}
$$

where $\cos ^{2} \xi$ and $\sin ^{2} \xi$ can obviously be expressed in terms of $\left(k_{x}, k_{\perp}\right)$. Note that the second and third angular terms in the last bracketed term are functions of $\Theta$ whereas the first of the terms is not. This will be an important point in the physical interpretation of this expression.

Equation (11) can be simplified by introducing variables $(u, v)$ such that $k_{x} \equiv k_{\mathrm{o}} u, k_{\perp}=k_{\mathrm{o}} v$, with $1 \leq u \leq \frac{k_{\mathrm{i}}}{k_{\mathrm{o}}}$, $0 \leq v \leq \infty$. All dependence of the integrand on the magnitude of the wavenumber passes through the integrals, leaving the integrations over the dimensionless parameters $u$ and $v$. Further simplification can be had by introducing an angular variable $\zeta$ defined as $\tan \zeta \equiv \frac{v}{u}$. This simplification allows the integral over $u$ to be done analytically, leaving the integrals over $\zeta$.

The resultant expression for the dissipation rate is then

$\epsilon_{N}=\sqrt{\frac{\pi}{8}} C_{B}^{2} F(\beta) V_{\mathrm{A}} 3 k_{\mathrm{i}}^{1 / 3} \Gamma_{1}(\Lambda)\left[T_{I}+T_{\Theta} \sin ^{2} \Theta\right]$.

The quantities $\Gamma_{1}, T_{I}$, and $T_{\Theta}$ are functions of $\Lambda$, related to integrals over $\zeta$, and are defined as follows.

$$
\begin{aligned}
& T_{I} \equiv\left[1-\frac{\Gamma_{2}}{\Gamma_{1}}\right], \\
& T_{\Theta} \equiv\left[\frac{\Gamma_{2}}{\Gamma_{1}}-\frac{\Gamma_{3}}{2 \Gamma_{1}}\right], \\
& \Gamma_{1}(\Lambda) \equiv \int_{0}^{\pi / 2} \mathrm{~d} \zeta \frac{\sec ^{2} \zeta \tan \zeta}{\left[1+\Lambda^{2} \tan ^{2} \zeta\right]^{\delta}}, \\
& \Gamma_{2}(\Lambda) \equiv \int_{0}^{\pi / 2} \mathrm{~d} \zeta \frac{\sec \zeta \tan \zeta}{\left[1+\Lambda^{2} \tan ^{2} \zeta\right]^{\delta}},
\end{aligned}
$$


Table 1. Functions of Lambda.

\begin{tabular}{|c|c|c|c|c|c|c|c|}
\hline$\Lambda$ & $\Gamma_{1}$ & $\Gamma_{2}$ & $\Gamma_{3}$ & $T_{I}$ & $T_{\Theta}$ & $I$ & $R^{\prime}$ \\
\hline 2 & $2.416 \times 10^{-1}$ & $1.186 \times 10^{-1}$ & $1.230 \times 10^{-1}$ & $5.09 \times 10^{-1}$ & $2.36 \times 10^{-1}$ & $2.305 \times 10^{-1}$ & 1.874 \\
\hline 5 & $2.807 \times 10^{-2}$ & $2.217 \times 10^{-2}$ & $5.895 \times 10^{-3}$ & $2.10 \times 10^{-1}$ & $6.85 \times 10^{-1}$ & $9.884 \times 10^{-3}$ & 1.677 \\
\hline 10 & $6.365 \times 10^{-3}$ & $5.815 \times 10^{-3}$ & $5.499 \times 10^{-4}$ & $8.64 \times 10^{-2}$ & $8.70 \times 10^{-1}$ & $8.688 \times 10^{-4}$ & 1.580 \\
\hline 14.1 & $3.109 \times 10^{-3}$ & $2.944 \times 10^{-3}$ & $1.650 \times 10^{-4}$ & $5.31 \times 10^{-2}$ & $9.20 \times 10^{-1}$ & $2.548 \times 10^{-4}$ & 1.544 \\
\hline 20 & $1.532 \times 10^{-3}$ & $1.483 \times 10^{-3}$ & $4.895 \times 10^{-5}$ & $3.20 \times 10^{-2}$ & $9.52 \times 10^{-1}$ & $7.419 \times 10^{-5}$ & 1.514 \\
\hline
\end{tabular}

$\Gamma_{3}(\Lambda) \equiv \int_{0}^{\pi / 2} \mathrm{~d} \zeta \frac{\sec \zeta \tan ^{3} \zeta}{\left[1+\Lambda^{2} \tan ^{2} \zeta\right]^{\delta}}$.

Given a value of $\Lambda$, the quantities $\Gamma_{1}, \Gamma_{2}, \Gamma_{3}$ are just numbers, parameters to be used in Eq. (12). They were evaluated numerically with a Mathematica workbook. Values of $\Gamma_{1}, \Gamma_{2}, \Gamma_{3}, T_{I}$, and $T_{\Theta}$ are given in Table 1 for several values of $\Lambda$.

Before leaving this section, we briefly return to the speculation in Sect. 2, i.e. that a more plausible model for an obliquelypropagating fast mode spectrum would be one in which surfaces of constant spectral power lay on the surfaces of cones, rather the elliptical surfaces shown in Fig. 1. In such a case, it seems likely that a heating rate expression like (12) would result, but in which the $T_{\Theta}$ term would be larger relative to the $T_{I}$ term. The reason for this is that in such a conical model, all wavenumbers would be more tightly collimated along the $x$ direction. The point in bringing this up is to note that in other, perhaps more plausible 3D spectra, the original expressions (6) and (7) would be an even better approximation than the model power spectrum considered in this paper.

\section{Comparison of new expression for dissipation rate with previous expressions}

In this section, I compare Eq. (12), calculated by integrating $2 \gamma(\boldsymbol{k}) P_{B}(\boldsymbol{k})$ over the entire wavenumber space of Fig. 1, with the approximate formula used in Papers I and II, as well as the expression derived and advocated by LS.

Before doing this, it is worthwhile to note a physicallysignificant aspect of (12). There are two terms in the dissipation rate, one proportional to $\sin ^{2} \Theta$, the other independent of $\Theta$. The term proportional to $\sin ^{2} \Theta$ is similar in form to Eq. (7). The other term, proportional to $T_{I}$, describes dissipation even in the case of parallel propagation. This represents the first result of the present paper; contrary to what was presented in Papers I and II, parallel propagation of the wave ensemble does not turn off the wave heating, so the expressions used in Papers I and II underestimate heating of the interstellar medium by damping of fast mode waves. The physical reason for this may be easily appreciated from Fig. 1. Even when the systematic propagation angle $\Theta=0$, there are modes in the spectrum which are obliquely propagating, and which will therefore be damped, yielding their energy to the thermal energy of the plasma.

I first compare Eq. (12) with Eq. (7), which was the basis of the analysis in Papers I and II. Substituting (4) into (12), dedimensionalizing the Alfvén speed, rms magnetic field amplitude, and inner and outer scales as described following Eq. (7), and choosing $\Lambda=5$ for a concrete example, we have

$$
\begin{aligned}
\epsilon(\Lambda=5)= & {\left[3.08 \times 10^{-21}+1.00 \times 10^{-20} \sin ^{2} \Theta\right] } \\
& \times \frac{F(\beta) V_{\mathrm{A}}^{\prime} b_{\mathrm{T}}^{\prime 2}}{\left(l_{\mathrm{o}}^{\prime 2} l_{\mathrm{i}}^{\prime}\right)^{1 / 3}} \mathrm{ergs} / \mathrm{s} / \mathrm{cm}^{3} .
\end{aligned}
$$

Equation (18) has two terms, one proportional to $\sin ^{2} \Theta$ and identifiable with that used in Papers I and II, and an isotropic term. The term proportional to $\sin ^{2} \Theta$ is in excellent agreement with the formula used in Papers I and II. This shows that the expression utilized in our earlier calculation is not only acceptable as an approximation; it is a very good approximation.

A calculation carried out with a larger, and perhaps extreme, value of $\Lambda=20$ yields a term proportional to $\sin ^{2} \Theta$ in even better agreeement with Eq. (7), and an isotropic term which is smaller in magnitude. As the spectrum becomes more anisotropic, characterized by $\Lambda \rightarrow \infty$, the isotropic term becomes negligible compared to the $\sin ^{2} \Theta$ term.

As noted immediately above, the inaccuracy of the expression used in Papers I and II was the omission of the isotropic term. Thus the more exact expression presented here predicts greater heating of the interstellar gas by Landau damping, and exacerbates rather than remedies the theoretical difficulty referred to in those papers.

\subsection{Comparison with the Lerche \& Schlickeiser expression}

The expression of LS for the Landau damping heating rate is

$\epsilon_{\mathrm{LS}}=\sqrt{\frac{\pi}{2}} C_{B}^{2} F_{\mathrm{LS}}(\beta) V_{\mathrm{A}}\left[\frac{k_{\mathrm{i}}^{2-s}-k_{\mathrm{o}}^{2-s}}{2-s}\right] I\left(\mu_{\mathrm{L}}, \Lambda, s\right)$,

where

$I\left(\mu_{\mathrm{L}}, \Lambda, s\right) \equiv \int_{\mu_{\mathrm{L}}}^{1} \mathrm{~d} \mu \frac{\left(1-\mu^{2}\right)}{\mu\left[\mu^{2}+\Lambda^{2}\left(1-\mu^{2}\right)\right]^{\delta}}$,

and $\mu_{\mathrm{L}}$ is a lower limit to the cosine of the polar angle of the wavenumber, identified by LS as $V_{\mathrm{A}} / v_{\mathrm{e}}$, where $v_{\mathrm{e}}$ is the electron thermal speed. The function $F_{\mathrm{LS}}(\beta)$ is defined in Eq. (24) below, and denotes the form of the function $F(\beta)$ used by LS. Equations (19) and (20) are identical to Eq. (11) of LS, with the exception that $\Lambda^{2}$ is used in place of $\Lambda$, in accordance with my convention, as discussed in Sect. 2.

To compare the Lerche and Schlickeiser expression with that in Sect. 4, I set $s=5 / 3$ and utilize the (excellent for the interstellar medium) approximation that $\frac{k_{\mathrm{i}}}{k_{\mathrm{o}}} \gg 1$. The function $I\left(\mu_{\mathrm{L}}, \Lambda\right)$ (again, merely a number for the purposes of these 
calculations) was evaluated with a Mathematica workbook. Values of $I\left(\mu_{\mathrm{L}}, \Lambda\right)$ are given in Col. 7 of Table 1 . The net expression for the Lerche and Schlickeiser dissipation rate is then

$\epsilon_{\mathrm{LS}}=\sqrt{\frac{\pi}{2}} V_{\mathrm{A}} F_{\mathrm{LS}}(\beta) C_{B}^{2} 3 k_{\mathrm{i}}^{1 / 3} I\left(\mu_{\mathrm{L}}, \Lambda\right)$.

Clearly this is very similar in form to Eq. (12). To more closely assess the similarity or difference, we can form the ratio of the two expressions by dividing (19) by (12),

$R \equiv \frac{\epsilon_{\mathrm{LS}}}{\epsilon_{N}}=\frac{2 F_{\mathrm{LS}}(\beta) I(\mu, \Lambda)}{F(\beta) \Gamma_{1}(\Lambda)\left[T_{I}+T_{\Theta} \sin ^{2} \Theta\right]}$.

Equation (22) shows that the relative magnitude of the two expressions is determined by two factors: (1) the expression adopted for $F(\beta)$ and (2) the assumption about the form of the power spectrum.

It should be noted that the factor of 2 in the numerator of (22) is superfluous, and is an "artifact" of holding the parameter $C_{B}^{2}$ constant in expressions (12) and (19) rather than $b_{\mathrm{T}}^{2}$. The spectrum of LS is symmetric about $k_{x}=0$ whereas that of Fig. 1 populates wavenumber space only with modes having $k_{x}>0$. If the turbulent variance $b_{\mathrm{T}}^{2}$ were held constant in the two expressions, this factor of 2 would not be present.

In comparing the expressions, I also set $\Theta=0$ since LS did not consider the possibility of oblique propagation. I therefore consider the parameter $R^{\prime} \equiv \frac{I}{\Gamma_{1} T_{I}}$ which ignores the aforementioned factor of 2 , and also isolates the effect of the assumptions about the form of the power spectrum from issues regarding the value of $F(\beta)$, which is a different matter (see next section). The values of $R^{\prime}$ are listed in Col. 8 of Table 1 . The values range from a maximum of 1.87 for the case of slight anisotropy (where the derivation of Sect. 4 begins to be invalid) to an apparently asymptotic value of $1.5-1.6$ for $\Lambda \geq 10$. Thus the two expressions, Eq. (12) and that of LS agree to within a small, and astrophysically negligible factor which can plausibly be attributed to minor differences in the way the integration over $\mathrm{k}$ space is performed, i.e. for $k_{x} \geq k_{\mathrm{o}}$ or $|k| \geq k_{\mathrm{o}}$.

\section{The dependence of the dissipation rate on the plasma $\beta$}

The function $F(\beta)$ describes the dependence of the Landau damping rate, and thus the wave heating rate, on the plasma $\beta$, i.e. on the temperature of the electrons and ions, and how they resonate with the fast mode waves.

Papers I and II as well as LS use the following formula for the Landau damping rate, taken from Ginzburg (1961)

$$
F(\beta)=\left(\frac{v_{\theta i}}{V_{\mathrm{A}}}\right)^{2}\left(\frac{V_{\mathrm{A}}}{v_{\mathrm{e}}}\right)+5\left(\frac{v_{\theta i}}{V_{\mathrm{A}}}\right) \exp \left[-\frac{V_{\mathrm{A}}^{2}}{2 v_{\theta i}^{2} \cos ^{2} \theta}\right]
$$

where $v_{\theta i}$ is the ion thermal speed and $v_{\mathrm{e}}$ is the electron thermal speed. Henceforth, I use the parameter $x \equiv v_{\theta i} / V_{\mathrm{A}}$ as a proxy for $\beta$. The function $F(\beta)$ monotonically rises with $x$, reaching $F \simeq 1$ for $x \simeq 0.7$ and $\theta \simeq 0$.

In Paper I, $F(\beta)$ was set to unity for the calculations, since $x$ is of order 1 in the interstellar medium. In Paper II, more specific plasma parameters were used, based on improved and
Table 2. Plasma properties of the diffuse interstellar gas.

\begin{tabular}{lll}
\hline \hline Variable & Value & Units \\
\hline$V_{\mathrm{A}}$ (Alfvén speed) & $2.33 \times 10^{6}$ & $\mathrm{~cm} / \mathrm{s}$ \\
$v_{\theta i}$ (ion thermal speed) & $0.93 \times 10^{6}$ & $\mathrm{~cm} / \mathrm{s}$ \\
$n_{\mathrm{e}}$ (plasma density) & 0.080 & $\mathrm{~cm}^{-3}$ \\
$B_{0}$ (mean magnetic field) & 3 & microGauss \\
$l_{\mathrm{o}}$ (outer scale of turbulence) & 3.6 & parsecs \\
$l_{\mathrm{i}}$ (inner scale of turbulence) & 800 & kilometers \\
$b_{\mathrm{T}}$ (amplitude of magnetic fluctuations) & 0.90 & microGauss \\
\hline
\end{tabular}

more accurate plasma measurements for the DIG (Minter \& Spangler 1996). These plasma quantities are listed in Table 2. These parameters were also used in the calculations of LS and will be used in the rest of this paper. LS assumed that the second term in Eq. (23) is negligible compared to the first. Given this assumption, which they contend is valid for all angles of propagation $\theta$, the effective $F(\beta)$ is

$F_{\mathrm{LS}}(\beta)=\frac{1}{V_{\mathrm{A}}}\left(\frac{v_{\theta i}^{2}}{v_{\mathrm{e}}}\right)=\frac{1}{42.9} x$.

With the last equality being true if the ion and electron temperatures are equal, as we assume. For the parameters of Table 2, Eq. (24) gives $F_{\mathrm{LS}}=9.3 \times 10^{-3}$, substantially different from $F \simeq 1$ assumed in Paper I.

The definitive discussion of Landau damping of fast mode waves is given in Barnes (1966). That paper presents numerical solutions to the Vlasov dispersion relation for a wide range of plasma propeties, so concern about the validity of approximations leading to formulas such as (5) and (23) is avoided. A major point of the discussion of Barnes (1966) is that the damping rate as a function of $\theta$ consists of two components, an ion peak and an electron peak, describing Landau resonance with ions and electrons, respectively. See for example, Figs. 3 and 8 of Barnes (1966). The assumption employed by LS in obtaining Eq. (24) appears to be be equivalent to neglecting the contribution of the ion peak.

Contrary to what is stated in LS, the ion peak contribution is not negligible for conditions appropriate to the DIG. Using data from Table 2 (specifically the values for $v_{\theta i}$ and $V_{\mathrm{A}}$ ), we find that the second term in Eq. (23) is 0.087 for the case of $\theta=0$, and 0.031 for $\theta=30^{\circ}$, leading to total $F$ parameters of $0.04-0.10$ for $\theta \leq 30^{\circ}$. Thus the heating rates of LS should be multiplied by a factor of several if applied to the DIG.

It should be conceded that the data of Table 2 are still averages over a large region of space, and that there are doubtlessly regions where the $x$ value is sufficiently low to make the electron peak the sole contributor to the damping rate. However, even in this case, as will be seen below, the heating rate will overwhelm the cooling capacity of the DIG. Furthermore, there could presumably also be regions where $x \sim 1$, in which case the wave heating would be even larger.

The accuracy of the analytic expression for the Landau damping rate in Eqs. (5) and (23) may be checked by reference to the full numerical solution of Barnes (1966). For example, the curve in Fig. 3 of Barnes (1966) can be shown to correspond 
to the case $x=0.5$, very similar to our case $(x=0.4)$ and compatible, within the errors, with the DIG. From Fig. 3, it may be seen that for the angular range $10^{\circ}-20^{\circ}$ the value of $F(\beta)$ is in quite good agreement with Eq. (23), including the contribution of the ion peak. The case plotted in Fig. 9 of Barnes (1966) corresponds to $x=0.22$, and in this case the approximation of LS is correct; only the electron peak term contributes significantly.

\subsection{A conjecture regarding distribution functions in the interstellar medium and the magnitude of Landau damping}

A striking feature of $F(\beta)$ is the very strong dependence on the plasma $\beta$, or in terms of our variables, $x$. This is a consequence of the assumed Maxwellian distribution function for the ions, which results in the Gaussian function in (23). For sufficiently low $\beta$ or $x$, there are very few ions in the Maxwellian tail that can resonate with the fast mode waves.

Experience from observations of plasmas in the solar wind show ion distributions which generally depart significantly from Maxwellians, in that there is an enhanced tail (e.g. Marsch 1991; Marsch 1999, and references therein). These distributions will have many more resonant ions that would a comparable Maxwellian (as defined by temperature of the core distribution) and thus higher damping than predicted by the formulas in this paper. Although we do not presently have enough information on the microphysics of the DIG, it certainly seems plausible that the ion distribution functions in the DIG would be similar to those in the solar wind. In this case, $F(\beta)$ would be a much more gradual function of $\beta$ or $x$ than (23), so that $F(\beta)$ (and thus $\gamma_{d}$ ) would be larger at small values of $x$.

\section{The magnitude of turbulent dissipation and the heating rate in the DIG}

In this section, I return to the main topic of all the papers in this series, which is the heat input rate to the gas of the interstellar medium by the Landau damping of MHD turbulence. I will utilize the expressions of LS as well as those presented in this paper.

The results are presented in graphical form in Fig. 2, which is a simplified version of Fig. 1 of Paper II. The volumetric heating rate is presented as the vertical coordinate. The medium-gray shaded area at the bottom of the graph indicates the heating rates that can be accommodated by the radiative cooling capacity of the DIG. The light-gray shaded area gives the plausible range of heating rates due to damping of turbulence by ion-neutral collisions. The close correspondence and overlap of these two shaded areas led to the suggestion that ion-neutral collisional damping is an important thermodynamic process in the DIG (Paper II, Minter \& Balser 1997).

The dissipation-heating rates discussed in the present paper are represented by horizontal lines. The solid lines are computed with Lerche and Schlickeiser formula, Eq. (21), and the dashed lines are given by Eq. (12). All of the fast mode damping rate calculations utilize the same values for the turbulence parameters $b_{\mathrm{T}}, l_{\mathrm{o}}, l_{\mathrm{i}}$, and $V_{\mathrm{A}}$. For the Lerche and Schlickeiser
DIG Heating and Cooling Rates

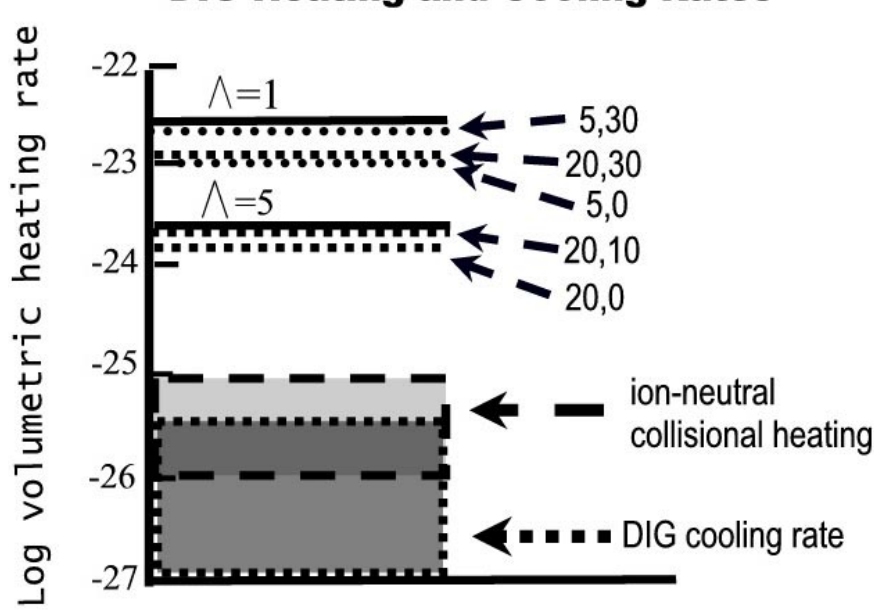

Fig. 2. Comparison of Landau Damping heating rates with the radiative cooling rate of the DIG. Shaded regions at the bottom indicate the radiative cooling rate and the heating rate due to ion-neutral collisional damping. Horizontal lines indicate heating rate due to the LS expression (solid lines) and that of the present paper (Eq. (12)) (dashed and dotted lines). The Lerche and Schlickeiser rates are calculated for values of the anisotropy parameter $\Lambda=1$ and 5 as indicated. The oblique wave model is calculated for values of $\Lambda=5$ and 20 (first number in label for heating rate) and for propagation angles $\Theta=0,10$, and 30 degrees (second number in label). This figure demonstrates that if the turbulence in the DIG consisted of fast mode magnetosonic waves, the heating rate would greatly exceed the radiative cooling rate.

expression, I utilized a value of $F_{\mathrm{LS}}(\beta)=9.3 \times 10^{-3}$, as adopted in LS, although as discussed in Sect. 6, it seems likely that this value is too low for the DIG. For the calculations utilizing our model for obliquely-propagating interstellar turbulence (Eq. (12)), I used $F(\beta)=0.040$. To simplify the calculation, I did not consider the $\theta$ dependence of $F(\beta)$ as may be seen in Eq. (21), but took a bulk value. This is obviously an approximation, but it is not likely to produce a significant quantitative difference.

The plotted values for the Lerche \& Schlickeiser expression adopt $\Lambda=1$ and $\Lambda=5$, as indicated. I consider these to be reasonable values for the ISM, but those are not the values advocated by Lerche and Schlickeiser. Further discussion of this crucial matter is given in Sect. 8 below. The oblique models were calculated for $\Lambda=5$ and $\Lambda=20$, and propagation angles of $0^{\circ}, 10^{\circ}$, and $30^{\circ}$, as indicated in the figure.

The conclusion to be drawn from Fig. 2 is that the heating rate due to dissipation of turbulence in the DIG exceeds the cooling capacity of that medium by a factor which ranges from just under 2 orders of magnitude to about three orders of magnitude. This statement obviously depends crucially and directly on the assumption that the interstellar MHD turbulence can be accurately described as an ensemble of fast mode waves. This statement was one of the principal conclusions of Papers I and II, as well as the articles cited there, and it is corroborated by the calculations of this paper. 


\section{The feasibility of highly anisotropic turbulence spectra}

The value for the turbulent dissipation rate claimed by LS for the DIG in Sect. 5.2 of their paper is actually quite close to the radiative cooling rate of $\simeq 3 \times 10^{-26} \mathrm{ergs} / \mathrm{s} / \mathrm{cm}^{3}$. However, the difference between that result and the calculations in Fig. 2 is not due to an artifact of the way the integration is carried out over wavenumber space (the topic of Sect. 4), nor of the value of $F(\beta)$ (the topic of Sect. 6) as was implied by those authors. LS obtained a heating rate $\simeq 3 \times 10^{-26} \mathrm{ergs} / \mathrm{s} / \mathrm{cm}^{3}$ by choosing an extreme value of $\Lambda^{2}=7400, \Lambda=86$ for the anisotropy of the turbulence.

There are two remarks to be made about the assumption of such a large anisotropy factor. First, the potential of hidden factors to drastically reduce the heating rate had already explicitly been made in Paper I. For example, in the last paragraph of Sect. 4.1.1, I said "The dependence of the linear Landau damping rate on $\theta(\Theta$ in the present terminology) and $F(\beta)$ should be particularly noted. If the interstellar MHD waves are either Alfvén waves or parallel-propagating fast mode waves, the linear Landau damping will be identically zero. The damping rate also depends strongly on the value of the plasma $\beta$. In a low- $\beta$ plasma linear Landau damping is greatly diminished. In conclusion, one can easily imagine circumstances under which the heating rate could be lowered by orders of magnitude". Lerche \& Schlickeiser note that for a sufficiently large anisotropy factor $\Lambda$, the heating rate can be adjusted to arbitrary and low values, but in Paper I it was noted that arbitrarily low values, including zero, could be accommodated by assuming pure parallel propagation.

The second remark is that such large anisotropies, while not unphysical, seem highly improbable for turbulence which is generated by naturally-occurring processes. In support of this scepticism, existing information is summoned on anisotropy of turbulence in other astrophysical plasmas.

\subsection{Measured anisotropy of interstellar turbulence}

Spangler \& Cordes (1998) summarized measurements of the anisotropy parameter $\Lambda$ for interstellar turbulence that existed up to that time, largely deduced from radio interferometric angular broadening measurements. These measurements show $1.0 \leq \Lambda \leq 5$. As discussed in that paper, it is possible that the true anisotropy factors are larger than the observed values, but by the same token, there is no evidence for $\Lambda \gg 5$.

\subsection{Coronal turbulence}

Radio propagation measurements have shown similar, but larger anisotropy factors in the density turbulence close to the Sun (heliocentric distances of 5-15 $R_{\odot}$, Armstrong et al. 1990; Grall et al. 1997). The measured values of $\Lambda$ range from $<5$ to as high as 15 . Even the highest values observed for coronal turbulence fall far short of the values suggested by LS. Furthermore, these high values are observed in parts of the corona where the $\beta$ is very low, unlike the DIG of the interstellar medium. In addition, the irregularities are spatially extended along the magnetic field, rather than perpendicular to it as the case presented here. Finally, it seems likely that this turbulence is better describable as quasi-two dimensional turbulence rather than propagating waves like fast mode magnetosonic waves.

\subsection{Turbulence near the Earth's bow shock}

The model of interstellar turbulence adopted in Paper I and continued here was inspired by the large amplitude MHD waves upstream of the Earth's bow shock. These waves have many observable characteristics which closely resemble the theoretical ideal of plane MHD waves. However, even for these waves the observed properties indicate that the modulation scale transverse to the propragation direction is several wavelengths, not tens or hundreds of times the wavelength (Spangler et al. 1988). This result was supported by the dual spacecraft measurements of Le \& Russell (1990), who found a transverse correlation length of about one Earth radius, which is of order the wavelength of these waves. The observations of the fast magnetosonic waves upstream of the Earth's bow shock indicate a value of $\Lambda \sim 5$ is plausible, and $\Lambda \leq 20$ worth considering as an extreme value. However, the data do not support larger anisotropy factors.

Obviously the turbulence in the Diffuse Ionized Gas (DIG) of the interstellar medium could be different than those plasmas discussed above. However, I feel that the proposal of extreme anisotropy is argued against by the fact that known turbulent space plasmas exhibit no such anisotropy.

In concluding this section, and indeed the presentation of the paper prior to the conclusions, it is appropriate to acknowledge the limits of our knowledge of interstellar turbulence, and the consequences of those limitations for the subject at hand. Although the basic plasma parameters of the DIG are well determined, they are nonetheless spatial averages obtained from line-of-sight integrations. It is possible that isolated regions could have properties quite different from the averages listed in Table 2, with important consequences for turbulent processes.

Our knowledge of the turbulence which occupies the DIG is even more uncertain. Models for the interstellar turbulence range from the MHD wave field discussed in this paper to the eddies described by two dimensional magnetohydrodynamics (Spangler 1999, and references therein). Given these limitations, one could plausibly postulate a central thermodynamic role for turbulence, and explore the circumstances under which there could be a balance between turbulent dissipation and radiative cooling of the Diffuse Ionized Gas. The discussion of LS can be considered such an exploration, and it identifies one way in which the substantial difference between estimated heating and cooling rates can be eliminated.

Nonetheless, the point of the present paper is that for this to be the case, the interstellar turbulence must have distinctive, and perhaps extreme properties. It would appear to be incumbent on those advocating such a thermodynamic balance to propose observational tests which could confirm or falsify the existence of such distinctive properties in interstellar turbulence. 


\section{Summary and conclusions}

The following are my conclusions about the magnitude of heating of the DIG by dissipation of turbulent fast magnetosonic waves within the context of the recent paper by Lerche \& Schlickeiser (2001).

1. Lerche \& Schlickeiser are entirely correct that, properly calculated, the dissipation rate is given by an integration over all $\boldsymbol{k}$ space, with the integrand being $2 \gamma_{d}(\boldsymbol{k}) P_{B}(\boldsymbol{k})$. Those contemplating work in this area should carry out the calculations correctly in this way.

2. Contrary to the assertion of LS, the expression derived in Paper I, involving a $1 \mathrm{D}-\mathrm{k}$ space integration over the reduced power spectrum is a good approximation for highly anisotropic, obliquely-propagating fields of fast mode waves, and is an acceptable approximation even when the anisotropy is moderate to small. The primary error involved in the equation of Papers I and II is that it underestimates heating due to Landau damping, in that an isotropic heating term is omitted.

3. The calculations in this paper support the conclusions of Papers I and II (and earlier works cited in those papers) that the heating rate of the DIG due to Landau damping exceeds, by two to three orders of magnitude, the cooling capacity of the interstellar medium. This statement is contingent on the DIG turbulence being primarily composed of fast magnetosonic waves (see next point). The heating rate can be reduced to the radiative cooling rate only if these waves are posited to have extreme or unlikely characteristics, such as $\Lambda \gg 1$.

4. I repeat here the conclusion of Papers I and II in this matter. Given these calculations, it seems that the Diffuse Ionized Gas has a very low energy density in fast mode magnetosonic waves. The well-observed turbulence in the DIG, and other parts of the interstellar medium, must consist of other types of magnetohydrodynamic excitations, such as a slightly compressible version of quasi-two dimensional turbulence. Such types of turbulence would not be subject to Landau damping as described above. One is then driven to conclude, as emphasized by E. Vazquez-Semadini in his review of Paper II, that a major issue in interstellar plasma physics is to understand how the interstellar turbulence can evolve without generating a thermodynamically significant level of fast mode waves.

Acknowledgements. This work was supported at the University of Iowa by grant ATM99-86887 from the National Science Foundation.

\section{References}

Armstrong, J. W., Coles, W. A., Kojjima, M., \& Rickett, B. J. 1999, ApJ, 358, 685

Barnes, A. 1966, Phys. Fl., 9, 1483

Cesarsky, C. J. 1980, ARA\&A, 18, 289

Cox, D. P., \& Reynolds, R. J. 1987, ARA\&A, 25, 303

Ginzburg, V. I. 1961, Propagation of Electromagnetic Waves in Plasma (New York: Gordon \& Breach)

Grall, R. R., Coles, W. A., Spangler, S. R., Sakurai, T., \& Harmon, J. K. 1997, J. Geophys. Res., 102, 263

Haffner, L. M., Reynolds, R. J., \& Tufte, S. L. 1999, ApJ, 523, 223

Hoppe, M. M., Russell, C. T., Frank, L. A., Eastman, T. E., \& Greenstadt, E. W. 1981, J. Geophys. Res., 86, 4471

Le, G., \& Russell, C. T. J. Geophys. Res., 95, 10703

Lerche, I., \& Schlickeiser, R. 2001, A\&A, 366, 1008 (LS)

Marsch, E. 1991, in Physics of the Inner Heliosphere, Vol. II, ed. R. Schwenn, \& E. Marsch (Heidelberg: Springer-Verlag), 45

Marsch, E. 1999, Space Sci. Rev., 87, 1

McIvor, I. 1977, MNRAS, 178, 85

Minter, A. H., \& Spangler, S. R. 1996, ApJ, 458, 194

Minter, A. H., \& Spangler, S. R. 1997, ApJ, 485, 182 (Paper II)

Minter, A. H., \& Balser, D. S. 1997, ApJ, 484, L133

Narayan, R., \& Hubbard, W. B. 1988, ApJ, 325, 503

Russell, C. T., Childers, D. D., \& Coleman, P. J. 1971, J. Geophys. Res., 76, 845

Spangler, S., Fuselier, S., Fey, A., \& Anderson, G. 1988, J. Geophys. Res., 93, 845

Spangler, S. R. 1991, ApJ, 376, 540 (Paper I)

Spangler, S. R. 1997, in Nonlinear Waves and Chaos in Space Plasmas, ed. T. Hada, \& K. Matsumoto (Tokyo: Terra Scientific Pub. Co.), 171

Spangler, S. R., \& Cordes, J. M. 1998, ApJ, 505, 766

Spangler, S. R. 1999, ApJ, 522, 879 